EPJ Web of Conferences 37, 07001 (2012)

DOI: $10.1051 /$ epjconf/20123707001

C) Owned by the authors, published by EDP Sciences, 2012

\title{
The SuperB factory, physics potential and project status
}

\author{
Jaroslaw Wiechczynski ${ }^{\mathrm{a}}$ \\ Institute of Nuclear Physics Polish Academy of Sciences, Cracow, Poland
}

\begin{abstract}
The SuperB project is an international enterprise aiming at the construction of the high-luminosity asymmetric beam energy electron-positron accelerator, which would be located in the area of Rome. It would exploit several novel features allowing to achieve an unprecedented luminosities and to collect almost a hundred times more data than the current generation of "B factories". As for the leptonic colliders, it will maintain a clean, low-background experimental environment that is crucial for numerous measurements on the field of high energy physics.
\end{abstract}

\section{Introduction}

The Standard Model (SM) is a current theory of fundamental particles and their interaction, however, it cannot be considered as an ultimate theory. Hence, several concepts of its extensions has been proposed so far. The Large Hadron Collider (LHC) at CERN is one of the fully operational experiment dedicated for the searches of the New Physics (NP) at high energies via hadron-hadron collisions. SuperB is a high luminosity flavour physics experiment that will perform detailed studies mainly of the $B$ mesons decays and its major mission is to reveal the mystery of the matter-antimatter asymmetry observed in the Universe, which cannot be fully explained by the SM. The machine consists of the high-luminosity asymmetric beam energy electron-positron accelerator along with the respective system of detectors. The SuperB project, based on the high-precision technology, plans to extend the studies undertaken at first generation of so-called B-factories.

In the following sections, the motivation of the project will be given along with detailed description of the main physical goals. Then the accelerator and the detector of the SuperB experiment will be briefly presented followed by the information on the status of the project.

\section{Project Motivation}

The new SupeB factory is basically motivated by a successful operation of the last decade B Factories related to Belle [1] and BaBar [2] experiments operating on KEKB and PEP-II colliders, respectively. They achieved numerous prominent results, among which the most important ones was the observation of the direct CP Violation in the $B$ meson decays and qualitative progress in the test of the unitarity of the CKM matrix [3] with the precision of the order of $1 \%$. Furthermore, many rare $B$ decays have been observed for the first time (ex. $B \rightarrow D \tau v$ ). Other succesful studies involve discovery of charmonium mixing, qualitative progress on the field of $\tau$ physics and the evidence of many new states. Those achievements shed some light on the basic laws of the nature, but, on the other hand, raised new questions of possible extensions of the Standard Model.

As the SuperB factory is supposed to work 50-100 times faster then the previous similar experiments, it aims to collect $75 \mathrm{ab}^{-1}$ of data at the $\Upsilon(4 S)$ resonance. This would allow to achieve a better precision and reveal possible new phenomena related to the quoted topics. Apart from the enormous data sample, the SuperB offers two unique features: $80 \%$ longitudinal polarization for electron

\footnotetext{
a e-mail: Jaroslaw.Wiechczynski@ifj.edu.pl
}

This is an Open Access article distributed under the terms of the Creative Commons Attribution License 2.0, which permits unrestricted use, distribution, and reproduction in any medium, provided the original work is properly cited. 
beam and the possibility to work at different energy regimes. Both should give the access to the very interesting analysis (see next chapter). Hence, the research program of the SuperB collaboration is complementary to the one already realized at the LHC.

\section{Physics program}

The physics program of the SuperB project will involve numerous precise measurements, allowing to find potential discrepancies from the Standard Model. The perspective of recording bigger data sample is crucial mainly on the field of $\Upsilon(4 S)$ physics concerning the studies on $B$ meson decays produced via $e^{+} e^{-} \rightarrow \Upsilon(4 S) \rightarrow B \bar{B}$ process. As in the case of the B Factories, the determination of the sides and angles of the Unitarity Triangle will still be a main goal, however, the new technology of SuperB will allow to reach a precision an order of magnitude better, comparing with the existing experiments. In particular, it would be possible to reach the precision of $1 \%(5 \%)$ in the determination of respective $\left|V_{c b}\right|$ and $\left|V_{u b}\right|$ parameters from the inclusive measurements [4]. Any discrepancy from the CKM paradigm would lead to the indication of the New Physics.

There are many $B$ meson decays allowing to construct observables that are highly sensitive to different NP scenarios. This mainly concerns the decays with the neutral particles in the final state (eg. $B \rightarrow X_{s} \gamma, B \rightarrow \tau v_{\tau}, B \rightarrow K_{S}^{0} \pi^{0} \gamma$ etc.), some of them have been already observed. Since the possibilities of analysing such processes are very limited at LHC experiments, SuperB can play a major role offering a chance for significant improvement of such measurements.

SuperB project can also have a major impact on the field of spectroscopy, exploring the world of charmonium-like states like X(3872), Y(4260), Y(4360), Y(4660) etc. B factories discovered plenty of new states and many of them do not fit into conventional mesons or byrons. Most of the new $c \bar{c}$ state has been observed in only a single decay mode with a significance slightly exceeding $5 \sigma$. SuperB would perform much more detailed studies, also in the different decay channels. Furthermore, the natural expectation is to discover new states at SuperB, previously unseen due to limited statistics.

\section{1 $\Upsilon(5 S)$ and charm physics}

One of the advantage of the SupeB project will be the flexibility of the collider. Scan of the energy in the center-of-mass $(\mathrm{CM})$ system will give an opportunity to run not only at $\Upsilon(4 S)$ but also at different regimes like $\Upsilon(5 S)$ or $D \bar{D}$ thresholds.

Studies on $B_{s}$ mesons are obvious at LHC experiments, however, $e^{+} e^{-}$colliders were also succesful on this field, so the studies will be continued at SuperB. Due to clear environment of $e^{+} e^{-}$annihilation via $\Upsilon(5 S)$, one of possible highlights are measurements of the decays with the neutral particles in the final state like $B_{s} \rightarrow J / \Psi \eta, B_{s} \rightarrow K_{S}^{0} \pi^{0}$ etc. Potential golden channel would be $B_{s} \rightarrow \gamma \gamma$ whose branching fraction (BF) predicted by the SM is about $10^{-7}$, however, in some NP scenarios (eg. SUSY) it can be enhanced by the order of magnitude. Interesting field of studies would be also the measurement of the semileptonic asymmetry of the $B_{s}$. SuperB plans to collect the data sample of 1 $\mathrm{ab}^{-1}$ at the $\Upsilon(5 S)$.

Another energy regime that is planned for the SuperB is charm threshold, where the data sample of $0.5 \mathrm{ab}^{-1}$ is expected after a few months running at the luminosity of $10^{35} \mathrm{~cm}^{-2} \mathrm{~s}^{-1}$. The CM energy will correspond to the $\psi(3770)$ resonance decaying into pair of the neutral $D$ mesons. This gives a possibility for tagging the events by identifying one $D$ meson of such pair, so the other one can be reconstructed with relatively small background contamination. In addition, similar to the $\Upsilon(4 S) \rightarrow B \bar{B}$ process, the $D \bar{D}$ are produced in the state of quantum entanglement. Having access to the quantumcorrelated $D$ decays, strong phases like $\delta_{K \pi}$ and $\delta_{K \pi \pi}$ may be determined independently and used for mixing measurements at the $\Upsilon(4 S)$. Furthermore, the data collected at the $\psi(3770)$ threshold would give an opportunity to significantly improve the precision (up to $10^{-4}$ ) of the mixing parameters $x_{D}$ and $y_{D}$. Other potential highlights from the SuperB would be studies on CP violation in $D \bar{D}$ oscillation and searches for the rare $D$ decays like $D^{0} \rightarrow \mu^{+} \mu^{-}$. 


\section{$3.2 \tau$ physics and electroweak measurements}

Another important part of the SuperB physics program are the studies in the sector of the $\tau$ lepton and the measurements of electroweak neutral current. On both of these fields, the crucial feature of the SuperB project will be $80 \%$ longitudinal polarization of the electron beam.

The $e^{-}$polarization along with the high luminosity will allow to determine electroweak neutral current parameters with the precision similar to the one achieved by LEP, however, at SuperB such measurements can be performed for $\mathrm{Q}^{2} \approx(10.58 \mathrm{GeV})^{2}$. This energy point is very interesting, especially with respect to the $\sin ^{2} \theta_{W}$ which has never been determined for that region, where, according to the SM, its value should vary with energy. For such measurement one can utilize the studies on the left-right asymmetry (by using pairs of $\mu^{+} \mu^{-}, \tau^{+} \tau^{-}, c \bar{c}$ and $b \bar{b}$ ), which can be determined with relative small uncertainty (of the order of $1 \%$ ).

Main topics related to the $\tau$ physics at SuperB include searches for lepton-flavor-violating (LFV) decays, studies of the $\tau$ electric dipole moment (EDM), anomalous magnetic moment $(g-2)$ and investigations on $\mathrm{CP}$ and $\mathrm{T}$ violation.

Studies of the lepton-flavor-violating processes are considered to be one of the most promising tool to discover the New Physics and explain its nature. The LFV effect has negligible theoretical uncertainties and since it should scale quadratically with the lepton mass, the $\tau$ - the heaviest of the family and having many possible LFV decay modes - seems to be the most suitable lepton to search for this phenomena. The Standard Model predicts that the branching fractions of the LFV decays of the charged leptons should be negligibly small. For instance, for the decay $\tau \rightarrow l \gamma$ it is expected to be less then $10^{-54}$. However, in some NP scenarios like supersymetric extensions of the SM, such processes should be significantly enhanced, even by several orders of magnitude. In particular, the branching fraction for the most promising decay channels $\tau \rightarrow \mu \gamma$ and $\tau \rightarrow \mu \mu \mu$ should be of the order of $10^{-9}$ and $10^{-10}$, respectively, which is within the sensitivity of the SuperB [5]. Moreover, the unique feature of the polarization offers the possibility to construct the additional discriminating variable that will be crucial in the background suppression in the studies of $\tau$ LFV.

There is a known discrepancy for the $\mu g-2$ factor between the experimental measurement and the value predicted by the SM and this difference should scale with the squared mass of the lepton. Therefore, this discrepancy can be interpreted as an indication of the NP if the respective difference in $\tau$ anomalous moment is of the order of $10^{-6}$. For this reason the precise determination of $\tau g-2$ factor is needed. Data collected at SuperB can provide the desired resolution to perform such measurement, which can also benefit from the feature of the beam polarization.

Both $g-2$ and EDM of the $\tau$ influence the angular distribution and the polarization of the $\tau$ produced in the $e^{+} e^{-}$annihilation. The proposed measurements for SuperB would include the analysis of the transverse and longitudinal $\tau$ 's polarization from the angular distribution of its decay products, as well as the fitting the distribution of the polar angle of the single $\tau$ lepton. It has been estimated that using the predicted data sample of $75 \mathrm{ab}^{-1}$ the $g-2$ form factor can be measured with the resolution of the order of $10^{-6}[6]$.

The CP violation in the sector of charged lepton has not been observed so far. According to the prediction of the SM, the CP asymmetry should be vanishingly small. For instance, for the $\tau^{ \pm} \rightarrow$ $K_{S}^{0} \pi^{ \pm} v_{\tau}$ process it is considered to be about $10^{-12}$ [7]. On the other hand, some of the NP frameworks (eg. R-parity violating SUSY) expects the enhancement of such asymmetries up to $10^{-1}$. SuperB is going to study the $\tau$ charged-dependent asymmetry of the angular distribution of the hadronic system produced in $\tau \rightarrow K_{S}^{0} \pi v_{\tau}$. The expected experimental sensitivity significantly exceeds the current limits estimated by CLEO.

\section{Accelerator \& Detector}

The accelerator [8] for the SuperB experiment is an $e^{+} e^{-}$collider designed to operate at wide range of $\mathrm{CM}$ energy and with extremely high luminosity at the level of $10^{36} \mathrm{~cm}^{-2} \mathrm{~s}^{-1}$. The latter will be provided by a specific collision scheme that encompass highly squeezed beam distribution at the interaction point, asymmetric beams with the $\mathrm{CM}$ boost $\beta \gamma=0.28$ crossing at $66 \mathrm{mrad}$, small collision area, 


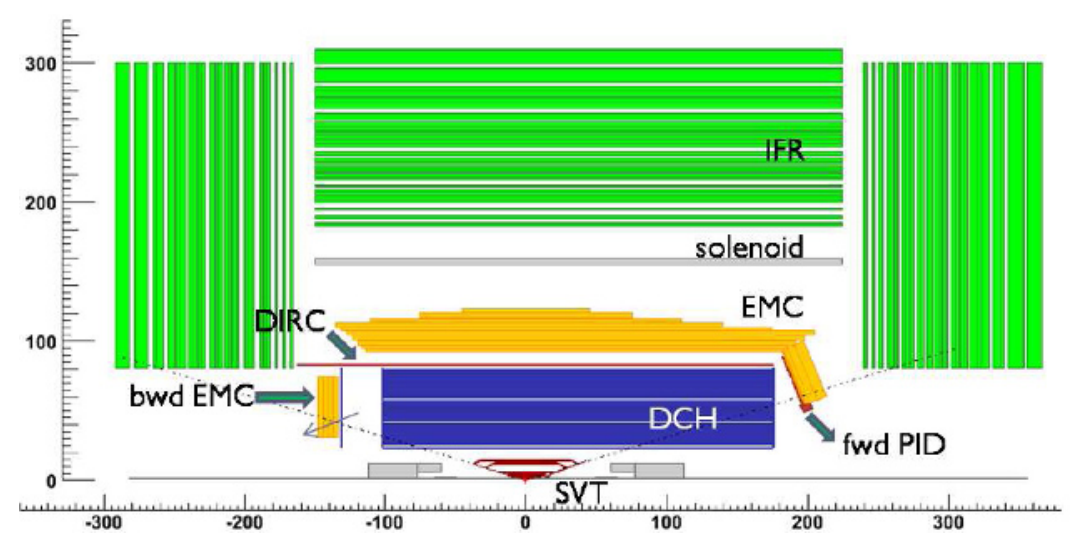

Fig. 1. Scheme of the SuperB detector.

large Piwinski angle and the "crab waist" transformation [9]. The last feature will be realized by two sextupoles magnets which turn one of the beam in a way that its waist is set to be perpendicular to the direction of the second beam. The "crab waist" concept will increase the luminosity by a factor of 2 with another advantage of the suppression of synchrobetatron resonances. The accelerator will be located on the campus of Tor Vergata University of Rome.

The idea of the SuperB detector [10] (Fig. 1) is based on the existing BaBar apparatus and will undergo some modifications that are necessary to operate at a designed luminosity and with a reduced center-of-mass boost. The tracking system of the detector consists of the silicon vertex detector (SVT) and a drift chamber $(\mathrm{DCH})$ inside a $1.5 \mathrm{~T}$ magnetic field. Particle identification of the SuperB detector is based on the concept of DIRC (Detector of Internally Reflected Cherenkov Light). The system of electromagnetic calorimeters (EMC) is built from three components: the barrel calorimeter (reused from $\mathrm{BaBar}$ and composed of $6580 \mathrm{CsI}(\mathrm{Tl})$ crystals), the forward calorimeter (from either pure CsI or LYSO crystals) and the respective backward endcap. The most external part of the SuperB apparatus is occupied by the instrumented flux return (IFR) detector. Its principal goal is to identify muons and, in conjunction with the electromagnetic calorimeter, to detect neutral hadrons such as $K_{L}^{0}$.

\section{Project Status}

During the last year, the project was fully approved, internationally recognized and received the major part of funding from the side of the Italian government. Moreover, the new laboratory, named after Nicola Cabibbo, was legally formed at Tor Vergata nearby to Rome to host the SuperB infrastructure. The data taking is scheduled to start in 2016/2018.

\section{References}

1. A. Abashian et al., (Belle Collab.), Nucl. Instrum. and Meth. A 479, 117 (2002).

2. B. Aubert et al., (BABAR Collab.), Nucl Instrum. Methods Phys. Res., Sect.A 479, 1 (2002).

3. N. Cabibbo, Phys. Rev. Lett. 10, 531 (1963). M. Kobayashi and T. Maskawa, Prog. Th. Phys. 49, 652 (1973).

4. SuperB Progress Reports, Physics, arXiv:1008.1541 (2010).

5. S. Antusch, E. Arganda, M.J. Herrero and A.M. Teixeira, JHEP 11, 090 (2006).

6. J. Bernabeu et al., Nucl. Phys. B 790160 (2008).

7. D. Delepine, G. Lopez Castro and L.T. Lopez Lozano, Phys. Rev. D 72, 033009 (2005).

8. SuperB Progress Reports, Accelerator, arXiv:1009.6178 (2010).

9. M. Zobov et al., arXiv:0802.2667 (2008).

10. SuperB Progress Reports, Detector, arXiv:1007.4241 (2010). 